\title{
Magnetic field fluctuation properties of coronal mass ejection-driven sheath regions in the near-Earth solar wind
}

Emilia K. J. Kilpua et al.

Correspondence to: Emilia K. J. Kilpua (emilia.kilpua@ helsinki.fi)

The copyright of individual parts of the supplement might differ from the CC BY 4.0 License. 


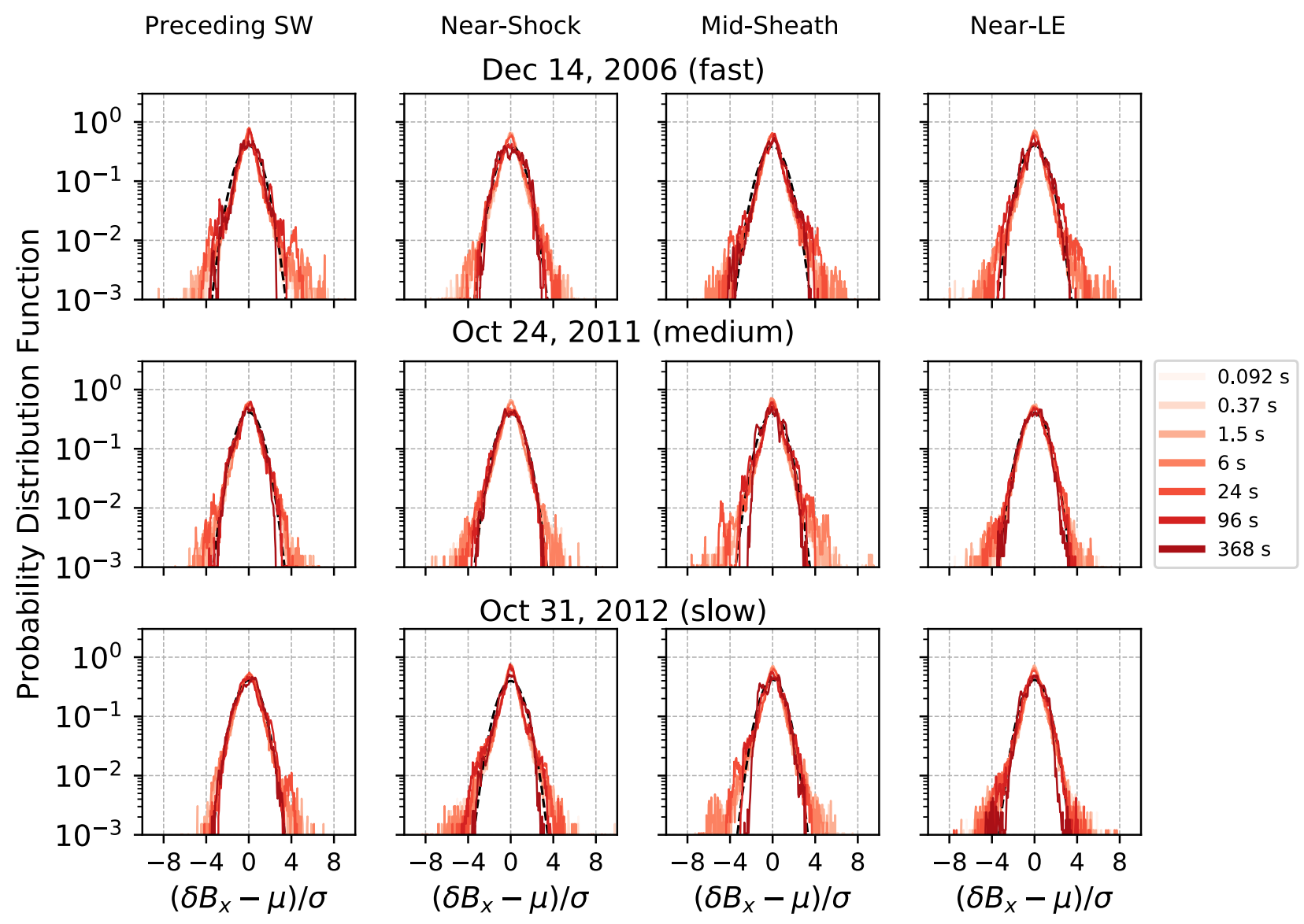

Figure S1. Probability distribution functions of for the magnetic field y-component in a similar format as Figure 4. 


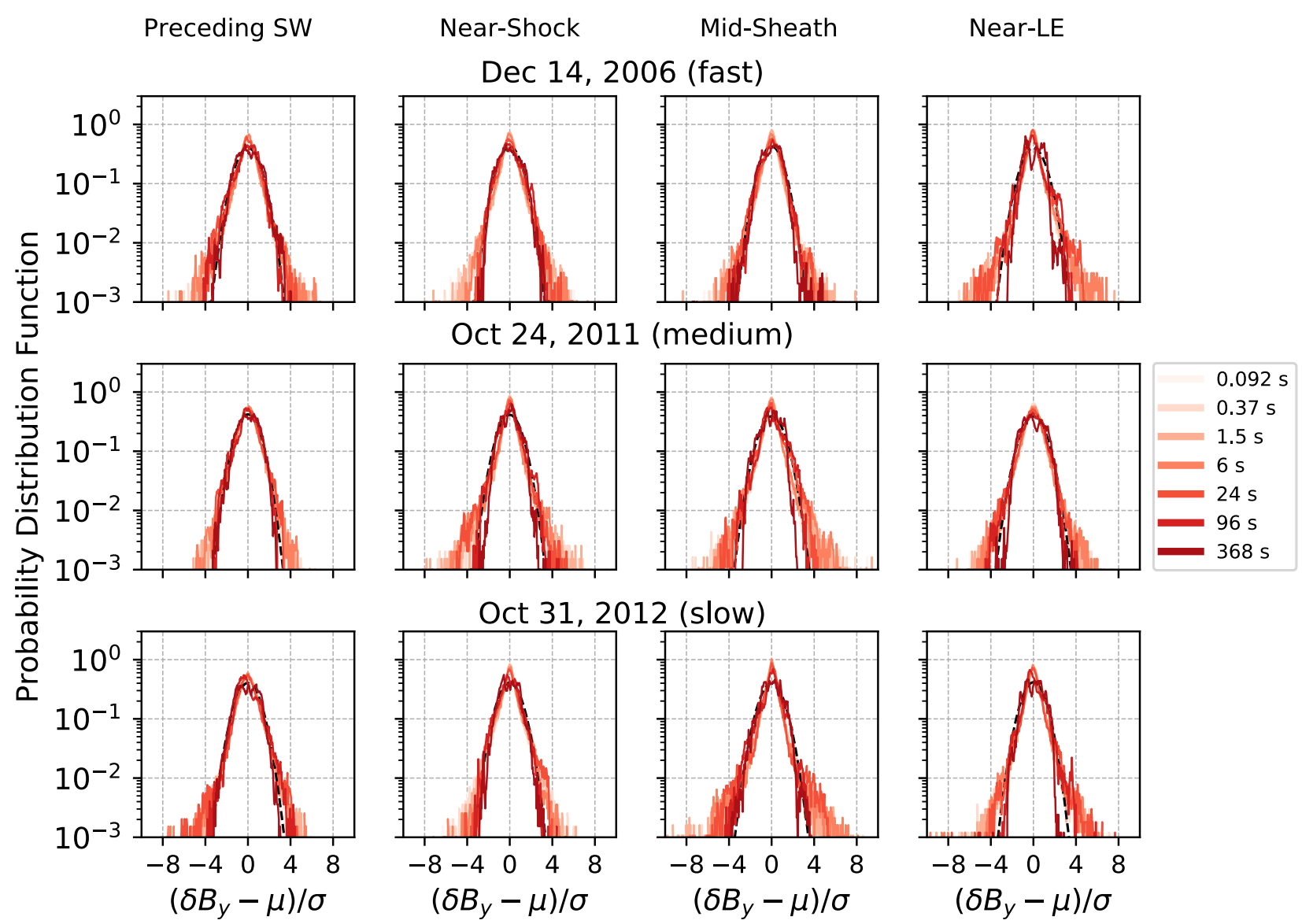

Figure S2. Probability distribution functions of for the magnetic field y-component in a similar format as Figure 4. 